\title{
NEURAL MESH ON CELLULAR NEURAL NETWORK
}

\author{
Tai-Hei Wu, Yng-Kae Tzeng, Cheng-Yuan Liou ${ }^{1}$ \\ Department of Computer Science and Information Engineering National Taiwan University \\ Taipei, Taiwan, R.O.C. \\ This work has been supported by NSC 93-2213-E-002-008. ${ }^{1} .{ }^{1}$ Correspondent, cyliou@csie.ntu.edu.tw
}

\begin{abstract}
This paper presents an implementation of the enural mesh on the cellular neural networks(CNN). An energy function is devised for the mesh to cope with the CNN. This energy can guide various behaviors of the mesh.
\end{abstract}

Index Terms-Hopfield model, cellular neural network, meshed snakes, elastic mesh, deformable mesh, surface modeling

\section{INTRODUCTION}

A mesh is designed to represent an image with a small number of nodes conveying most of the information about the image. Various meshes have been constructed for modeling objects with varying degrees of success, e.g., Weiss [15], Terzopoulos [6][11], Wang and Lee [14]. The mesh in [8] and [10] has been designed to emulate the soap film and solve the Steiner tree problem [3]. This mesh was further developed in 3D [9] to solve the minimal surface problem where the Potts technique was applied to minimize an energy of a 3D mesh. This paper presents an implementation of the neural mesh devised in [7] on the cellular neural network (CNN) [2] It follows the architecture of the CNN to implement the neural mesh. This paper reforms the energy function for the mesh and then minimizes this energy using the cellular neural network.

\section{REVIEW MESHED SNAKE}

\subsection{The Snake}

Kass et al. [6] proposed an energy-minimizing active contour model (named snake) which could drive a set of points to lie on features of interest, e.g. edges, in a image. The position of a snake point is represented by $v(s)=(x(s), y(s))$, where $(x(s), y(s))$ is the Cartesian coordinates in the image plane and $s$ is the distance along the snake spline. The energy function can be written as

$$
\begin{aligned}
E_{\text {snake }}^{*} & =\int_{0}^{1} E_{\text {snake }}(v(s)) d s \\
& =\int_{0}^{1}\left[E_{\text {int }}(v(s))+E_{\text {image }}(v(s))+E_{\text {con }}(v(s))\right] d s,
\end{aligned}
$$

where $E_{\text {int }}$ represents the internal energy of the spline due to bending or discontinuities, $E_{\text {image }}$ represents the image forces, and $E_{c o n}$ represents the external constrained forces. The internal spline energy can be written as:

$$
E_{\text {int }}=\left(\mu(s)\left|v_{s}(s)\right|^{2}+\nu(s)\left|v_{s s}(s)\right|^{2}\right) / 2 .
$$

In the above equation, the first-order term causes the snake to behave like a string to resist the stretching force and the second-order term causes the snake to behave like a rod to resist the bending moment. Adjusting their weights, $\mu(s)$ and $\nu(s)$, one can control the relative importance of the string and rod terms. The image force in the equation (1) comes from various events such as corners or edges.

Many methods have been proposed to minimize this energy function $[1,6,7,12,16]$. Liou and Chang [7] proposed an approach to evolve the mesh. Discretizing the equation (1) and ignoring $E_{c o n}$ for simplicity, the energy function can be written as

$E_{\text {snake }}=\sum_{i=1}^{n} \mu_{i}\left|v_{i}-v_{i-1}\right|^{2}+\nu_{i}\left|v_{i-1}-2 v_{i}+v_{i+1}\right|^{2}+E_{\text {image }}\left(v_{i}\right)$,

where $E_{\text {image }}\left(v_{i}\right)=-\gamma_{i}\left|\nabla I\left(v_{i}\right)\right|^{2}$ denotes the negated intensity gradient at point $v_{i}$.

The approach used a two-dimensional Hopfield neural network to minimize the equation (2). The network consists of $n * m$ mutually interconnected neurons where $n$ is the total number of points of the snake and $m$ is the total number of neighbors around each point of the snake. The energy function is computed at all $n$ snake points, $\left\{v_{i}, i=1, . ., n\right\}$, and their neighbors, as shown in Fig. 1. The neighbor position that minimizes the energy function is chosen as the new position of $v_{i}$. Let $v_{i, p}$ denote the binary state of the $(i, p)^{t h}$ neuron (1 for firing and 0 for resting). Let $x_{i, p}$ and $y_{i, p}$ be the $x$ and $y$ coordinates, respectively, of the neighboring point $(i, p)$, and let $g_{i, p}$ be the negated intensity gradient at the neighboring point $(i, p)$ defined as $g_{i, p}=-\gamma_{i}\left|\nabla I\left(x_{i, p}, y_{i, p}\right)\right|^{2}$. The Hopfield type energy function for the equation (2) is

(1) $E_{\text {snake }}=$

$$
\begin{aligned}
& \sum_{i=1}^{n}\left\{w _ { 1 } \alpha _ { i , i - 1 } \left[\left(\sum_{p=1}^{m} x_{i, p} v_{i, p}-\sum_{p=1}^{m} x_{i-1, p} v_{i-1, p}\right)^{2}+\right.\right. \\
& \left.\left(\sum_{p=1}^{m} y_{i, p} v_{i, p}-\sum_{p=1}^{m} y_{i-1, p} v_{i-1, p}\right)^{2}\right]+w_{2} \beta_{i-1, i+1}[
\end{aligned}
$$




$$
\begin{aligned}
& \left(\sum_{p=1}^{m} x_{i-1, p} v_{i-1, p}-2 \sum_{p=1}^{m} x_{i, p} v_{i, p}+\sum_{p=1}^{m} x_{i+1, p} v_{i+1, p}\right)^{2} \\
& \left.+\left(\sum_{p=1}^{m} y_{i-1, p} v_{i-1, p}-2 \sum_{p=1}^{m} y_{i, p} v_{i, p}+\sum_{p=1}^{m} y_{i+1, p} v_{i+1, p}\right)^{2}\right] \\
& +w_{3} \gamma_{i}\left(\sum_{p=1}^{m} g_{i, p} v_{i, p}\right)+w_{4} \varepsilon_{i}\left[\left(1-\sum_{p=1}^{m} v_{i, p}\right)^{2}\right] \\
& \left.+w_{5} \tau_{i} \sum_{p=1}^{m} v_{i, p}\left(1-v_{i, p}\right)\right\} .
\end{aligned}
$$

The fourth term of this energy function constrains that only one neighbor point can be selected from the neighboring points $(i, 1),(i, 2), \ldots,(i, m)$ for each $i$. The last term is called selfconnection eliminating term, which will reduce the diagonal value of the interconnection matrix of the Hopfield network. The $\mu_{i}$ and $\nu_{i}$ in equation (2) are replaced by $\alpha_{i, i-1}$ and $\beta_{i-1, i+1}$ respectively. $\alpha_{i, j}$ and $\beta_{i, j}$ are defined as

$$
\begin{gathered}
\alpha_{i, j}=1-\left(g_{i, 5}+g_{j, 5}\right) / 10 \\
\beta_{i, j}=\left(15+g_{i, 5}+g_{j, 5}\right) / 40
\end{gathered} .
$$

There are many other designs for the functions $\alpha_{i, j}$ and $\beta_{i, j}$. Different designs produce different results. The value of $g_{i, j}$ is normalized with a range from 0 to -255 . The five coefficient constants $w_{1}, w_{2}, w_{3}, w_{4}$ and $w_{5}$ are used to weight the five terms.

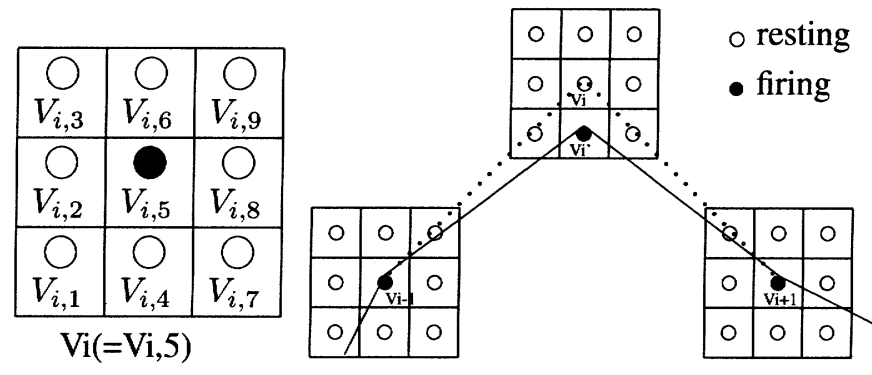

Figure 1. Position of a neuron. Each node in the snake has eight neighbors. Thus, $m=9$ in this case(including itself). The energy function is computed at $v_{i}$ and each of its eight neighbors. The location $v_{i}^{\prime}$, that minimizes the energy function, is selected as the new position of $v_{i}$.

\subsection{The Mesh}

The neural mesh in [7] is composed of many snakes, see Fig. 2(a). The energy function of the mesh is:

$$
E_{m e s h}=\sum_{s=1}^{S_{n}} E_{\text {snake }(s)} .
$$

The energy function of a two-deimensional Hopfield network is written as:

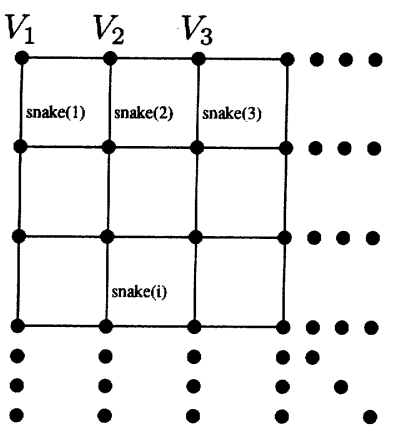

(a)

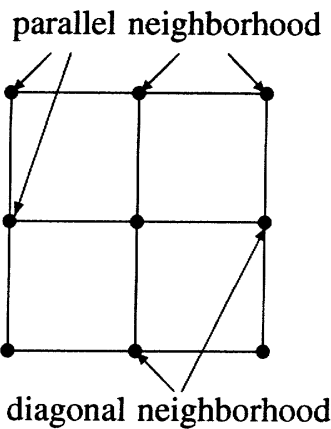

(b)
Figure. 2. Composition of neural and their relation. (a): The mesh is composed of many snakes. Each snake has four nodes. (b): The snake has a tendency to shrink if there is no constraint imposed upon it. Each internal nodes in the mesh is drived by eight forces.

$$
\begin{aligned}
E_{\text {Hopfield }}= & -\frac{1}{2} \sum_{i=1}^{N} \sum_{p=1}^{m} \sum_{j=1}^{N} \sum_{q=1}^{m} W_{i, p ; j, q} v_{i, p} v_{j, q} \\
& -\sum_{i=1}^{N} \sum_{p=1}^{m} I_{i, p} v_{i, p}
\end{aligned}
$$

where $N$ is the total number of nodes of the whole mesh.

Rearranging equation (5) and comparing it with the equation ((3) we obtain:

$$
\begin{aligned}
W_{i, p ; j, q}= & \left(T_{1}(i, j)+T_{2}(i, j)+T_{3}(i, j)\right) \\
& *\left(x_{i, p} x_{j, q}+y_{i, p} y_{j, q}\right)+T_{4}(i, j) .
\end{aligned}
$$

In the equation (6), the four terms are

$$
\begin{aligned}
T_{1}(i, j)= & \left(2-\delta_{p, q}\right) \delta_{i, j}\left[\sum_{k=1}^{N}-4 \phi_{2}(i, k)\left(w_{1} \alpha_{i, k}+w_{2} \beta_{i, k}\right)\right. \\
& \left.-8 \sum_{l=1}^{N} w_{2} \beta_{k, l} \phi_{1}(i, k) \phi_{1}(i, l) \phi_{2}(k, l)\right] ; \\
T_{2}(i, j)= & 8 w_{1} \alpha_{i, j} \phi_{2}(i, j) \\
T_{3}(i, j)= & 8 w_{2}\left\{\sum _ { k = 1 } ^ { N } \left[\beta_{i, k} \phi_{1}(j, k) \phi_{2}(i, k)+\right.\right. \\
& \left.\left.\beta_{j, k} \phi_{1}(i, k) \phi_{2}(j, k)\right] \phi_{1}(i, j)-\beta_{i, j} \phi_{2}(i, j)\right\} ; \\
T_{4}(i, j)= & {\left[\sum_{k=1}^{N}\left(-2 w_{4} \varepsilon_{i}\left(2-\delta_{p, q}\right)+2 w_{5} \tau_{i} \delta_{p, q}\right) \phi_{2}(i, k)\right] \delta_{i, j} ; } \\
I_{i, p}= & \sum_{k=1}^{N}\left[\left(-w_{3} \gamma_{i} g_{i, p}+2 w_{4} \varepsilon_{i}-w_{5} \tau_{i} \delta_{p, q}\right) \phi_{2}(i, k)\right.
\end{aligned}
$$

respectively. $\delta_{i, j}$ is the Kronecker delta function. $\phi_{1}(i, j)$ and $\phi_{2}(i, j)$ are the neighborhood functions defined as in Fig. 2(b), where 


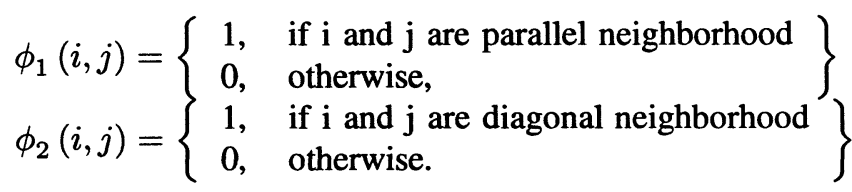

The $(i, p)^{\text {th }}$ neuron in the mesh network receives an input weighted by $W_{i, p ; j, q}$ from the $(j, q)^{t h}$ neuron, and a bias input $I_{i, p}$. The total input, net $_{i, p}$, of the $(i, p)^{t h}$ neuron is calculated as

$$
n t_{i, p}=\sum_{j=1}^{N} \sum_{q=1}^{m} W_{i, p ; j, q} v_{i, p}+I_{i, p}
$$

Then, the $(i, p)^{t h}$ neuron is updated as

$$
v_{i, p}=\left\{\begin{array}{ll}
1, & \text { if } \text { net }_{i, p} \geq 0 \\
0, & \text { if } n e t_{i, p}<0
\end{array}\right\}
$$

The rule described above for updating each neuron's state is applied in an asynchronous mode. This means that for a given time, only a single neuron (which is selected randomly) is allowed to update its output state.

\section{MESH ON CELLULAR NEURAL NETWORK}

L. O. Chua and L. Yang [2] proposed a novel circuit architecture, named cellular neural networks, that can perform parallel signal processing in real time. It shares the features of the neural network and cellular automata. Its local dynamic is feasible to realize the neural mesh that has constraint over the neuron's neighbors. This paper slightly modifies the energy function of mesh, equation (3), to cope with the architecture of the cellular neural network.

We implement the mesh with $m * n$ points using two parallel and size-equaled cellular neural networks, denoted as $C_{x}$ and $C_{y}$, with $m * n$ neurons arranged in $m$ rows and $n$ columns. Let $c(i, j)$ denote the $(i, j)^{t h}$ neuron in this cellular neural network and $c_{N r}(i, j)$ denote the neighbors of $c(i, j)$. We include the four parallel neighbors of $c(i, j)$ in $c_{N r}(i, j)$. We reorganize the energy function of the mesh that has the CNN character. The neuron ouputs are either -1 or 1 . The new energy function is

$$
\begin{aligned}
& E_{\text {int }_{x}}=\sum_{c(i, j) \in C_{x}} \sum_{c(k, l) \in c_{N}(i, j)}\{ \\
& \alpha_{i, j}\left[\left(x_{i, j}+v_{x, i, j}-x_{k, l}\right)^{2}\right]+ \\
& \left.\sum_{\substack{c(p, q) \in c_{N r}(i, j) \\
c(p, q) \notin c_{N r}(k, l)}} \beta_{i, j}\left[\left(x_{k, l}-2\left(x_{i, j}+v_{x, i, j}\right)+x_{p, q}\right)^{2}\right]\right\} \\
& E_{\text {image }_{x}}=\sum_{c(i, j) \in C_{x}} \gamma_{i, j}\left[\begin{array}{c}
g_{-1, x, i, j}\left(1-\left(v_{x, i, j}+1\right)^{2}\right) \\
+g_{+1, x, i, j}\left(1-\left(v_{x, i, j}-1\right)^{2}\right)
\end{array}\right] \\
& E_{\text {mesh }_{x}}=E_{\text {int }_{x}}+E_{\text {image }_{x} .}
\end{aligned}
$$

where $v_{x, i, j}$ is the output of $c(i, j) \in C_{x} . x_{i, j}, y_{i, j}$ and $g_{a, x, i, j}$ are the coordinates and negative intensity gradient, respectively, of the $(i, j)^{t h}$ neuron in mesh. $g_{a, x, i, j}$ is defined as $g_{a, x, i, j}=-\gamma_{i, j}\left|\nabla I\left(x_{i, j}+a, y_{i, j}\right)\right|^{2}$ and $g_{a, y, i, j}$ is defined as $g_{a, y, i, j}=-\gamma_{i, j}\left|\nabla I\left(x_{i, j}, y_{i, j}+a\right)\right|^{2}$. The $E_{\text {int }_{y}}$ and $E_{\text {image }_{y}}$ in the energy function $C_{y}$ are defined similarly by replacing all $x$ with $y$ in the formulas of $E_{i n t_{x}}$ and $E_{\text {image }}$. In the following, we discuss the case for $C_{x}$ only. The values of $v_{x, i, j}$ and $v_{y, i, j}$, outputs of $c(i, j) \in C_{y}$, are used to update the position of the $(i, j)^{t h}$ neuron in the mesh. The new position is

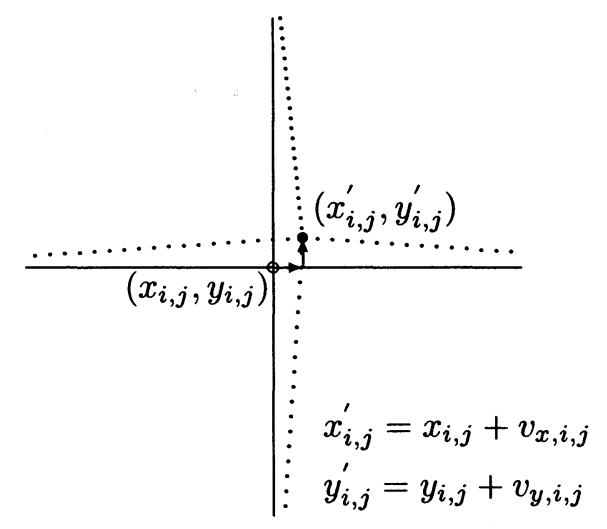

Figure. 3. The movement of a mesh node. The outputs of $C_{x}$ and $C_{y}$ serve as the movement of mesh nodes. Since the energy functions of $C_{x}$ and $C_{y}$ are minimized separately, coordinates $x_{i j}$ and $y_{i j}$ are updated in turn, not synchronously.

$$
\begin{aligned}
& x_{i, j}^{\prime}=x_{i, j}+v_{x, i, j} \\
& y_{i, j}^{\prime}=y_{i, j}+v_{y, i, j} .
\end{aligned}
$$

One may think of $v_{x, i, j}$ and $v_{y, i, j}$ as the movement of the $(i, j)^{t h}$ neuron in the mesh. The Lyapunov function of a cellular neural network with $m * n$ neurons is

$$
\begin{aligned}
E_{c n n}= & -\frac{1}{2} \sum_{(i, j)} \sum_{(k, l)} A(i, j ; k, l) v_{i, j} v_{k, l} \\
& -\sum_{(i, j)} \sum_{(k, l)} B(i, j ; k, l) v_{i, j} v_{u, k, l} \\
& +\frac{1}{2 R_{x}} \sum_{(i, j)} v_{i, j}^{2}-\sum_{(i, j)} I v_{i, j},
\end{aligned}
$$

where $R_{x}$ is an adjustable parameter, $v_{i, j}$ and $v_{u, i, j}$ are the output and input of the $(i, j)^{\text {th }}$ neuron in the cellular neural network. Let $I, A(i, j, k, l)$ and $B(i, j, k, l)$ be zero for all $i, k<m$ and $j, l<n$ when $i \neq k$ and $j \neq l$. Let $v_{u, i, j}$ be 1 for all $i<m$ and $j<n$. The Lyapunov function has a more simple form, that is

$$
\begin{aligned}
E_{c n n}^{\prime}= & -\frac{1}{2} \sum_{(i, j)} A(i, j ; i, j) v_{i, j}^{2}+\frac{1}{2 R_{x}} \sum_{(i, j)} v_{i, j}^{2} \\
& -\sum_{(i, j)} B(i, j ; i, j) v_{i, j}
\end{aligned}
$$




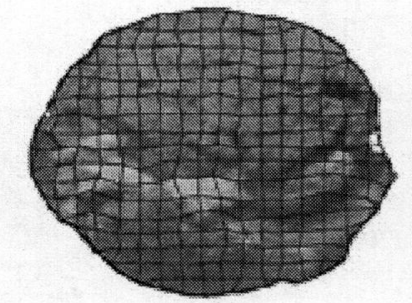

Figure 4. The final mesh.

In this way, the Lyapunov function of $C_{x}$ is

$$
\begin{aligned}
E_{C_{x}}= & -\frac{1}{2} \sum_{(i, j)} A_{x}(i, j ; i, j) v_{x, i, j}^{2}+\frac{1}{2 R_{x x}} \sum_{(i, j)} v_{x, i, j}^{2} \\
& -\sum_{(i, j)} B_{x}(i, j ; i, j) v_{x, i, j} .
\end{aligned}
$$

Rearranging and comparing $E_{m e s h_{x}}$ to $E_{C_{x}}$, we obtain

$$
\begin{aligned}
& A_{x}(i, j ; i, j)=\frac{1}{R_{x x}}-8 \alpha_{i, j}-32 \beta_{i, j} \\
& +2 \gamma_{i, j}\left(g_{-1, x, i, j}+g_{+1, x, i, j}\right) \\
& B_{x}(i, j ; i, j)= \\
& \sum_{c(k, l) \in c_{N r}(i, j)} \alpha_{i j} 2\left(x_{k, l}-x_{i, j}\right)+ \\
& \sum_{c(k, l) \in C_{x}} \sum_{c(p, q) \in C_{x}} 4 \beta_{i, j}\left(x_{k, l}+x_{p, q}-2 x_{i, j}\right) \\
& * \phi_{1}(i, j ; k, l) \phi_{1}(i, j ; p, q) \phi_{2}(k, l ; p, q) \\
& +2 \gamma_{i, j}\left(g_{-1, x, i, j}-g_{+1, x, i, j}\right),
\end{aligned}
$$

where $\phi_{1}(i, j ; k, l)$ and $\phi_{2}(i, j ; k, l)$ are the neighborhood functions redefined as

$$
\begin{aligned}
& \phi_{1}(i, j ; k, l)=\left\{\begin{array}{ll}
1, & \text { if } c(i, j) \text { and } c(k, l) \text { are parallel } \\
\text { neighborhood } & \text { otherwise }
\end{array}\right\} \\
& \phi_{2}(i, j ; k, l)=\left\{\begin{array}{ll}
1, & \text { if } c(i, j) \text { and } c(k, l) \text { are diagonal } \\
0, & \text { neighborhood }
\end{array}\right\}
\end{aligned}
$$

$A_{y}(i, j ; i, j)$ and $B_{y}(i, j ; i, j)$ for $C_{y}$ can be obtained similarly.

After setting $A_{x}(i, j ; i, j), B_{x}(i, j ; i, j), A_{y}(i, j ; i, j)$ properly [2], we obtain $C_{x}$ and $C_{y}$. We then apply $C_{x}$ and $C_{y}$ to obtain $v_{x, i, j}$ and $v_{y, i, j}$ for the new position of the $(i, j)^{t h}$ neuron in the mesh.

The $x$ and $y$ coordinates can be updated in turn in an asynchronous mode. One cellular neural network is sufficient for implementing the mesh. This means that at a given time only one of the $x$ or $y$ coordinates is updated to a new value but not both. The experimental results are shown in Fig. 4 and 5.

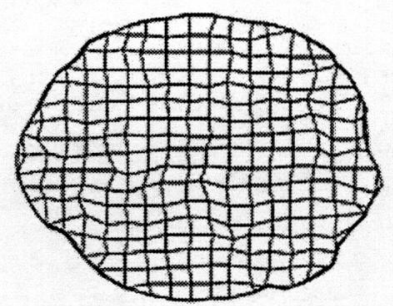

Figure 5. The coverged mesh only.

\section{SUMMARY}

This paper implemented the neural mesh on the cellular neural network. The implementation is to employ the cellular neural network to solve the energy-minimizing problem for the mesh. There are many ways to construct the energy function on the cellular neural network for the mesh, e.g., the mesh in [7] uses nine neurons for each mesh point. This paper devises an elegant one that uses only one cell in one cellular neural network for each mesh point.

\section{REFERENCES}

[1] A. A. Amini, S. Tehrani, and T. E. Weymouth "Using dynamic programming for minimizing the energy of active contours in the presence of hard constraints". Proceedings of the Second International Conference on Computer Vision. Tarpon Springs, Florida, Dec. 1988, pages 95-99.

[2] L. O. Chua and L. Yang "Cellular neural network: Theory". IEEE Transactions on Circuits and Systems, 35(10):1257-1272, 1988.

[3] E. N. Gilbert and H. O. Pollak "Steiner minimal trees". SIAM Journal on Applied Mathematics, 16:1-29, 1968.

[4] J. J. Hopfield and D. W. Tank "Neural computation of decisions in optimization problems". Biological Cybernetics, 52:141-152, 1985.

[5] F. K. Hwang and D. S. Richards "Steiner tree problems". Network, 22(1):55-89, 1992.

[6] M. Kass, A. Witkin and D. Terzopoulos "Snakes: Active contour models". Proceedings of the First international Conference on Computer Vision. London, England, Jun. 1987, pages 259-268.

[7] C. Y. Liou and Q. M. Chang "Meshed snakes". Proceedings of International Conference on Neural Network, Washington D. C., USA, Jun.1996, vol.3, pages 1516-1521.

[8] C. Y. Liou and Q. M. Chang "Numerical soap film for the steiner tree problem". Proceedings of International Conference on Neural Information Processing, Hong Kong, Sep. 1996, vol. 1, pages 642-647.

[9] C. Y. Liou and Q. M. Chang "Active mesh for minimal surface problems". Proceedings of International Conference on Neural Information Processing, Dunedin, New Zealand, Nov. 1997, vol. 1, pages 486-489.

[10] C. Y. Liou and Q. M. Chang "Neural mesh for the steiner tree problem". Journal of Information Science and Engineering, 13(2):335-354, 1997.

[11] D. Terzopoulos and M. Vasilescu "Sampling and reconstruction with adaptive meshes". Proceedings of IEEE International Conference on Computer Vision and Pattern Recognition, Lahaina, Maui, Hawaii, Jun. 1991, pages $70-75$

[12] C. T. Tsai "Minimizing the energy of active contour model using a Hopfield network". IEE Proceeding-E: Computers and Digital Techniques, 140(6):297-303, 1993.

[13] M. Vasilescu and D. Terzopoulos "Adaptive meshes and shells". Pro ceedings of IEEE International Conference on Computer Vision and Pattern Recognition, Champaign, Illinois, USA, Jun. 1992, pages 829831.

[14] Y. Wang and O. Lee "Active mesh - A feature seeking and tracking image sequence representation scheme". IEEE Transactions on Image Processing, 3(5):610-624, 1994.

[15] I. Weiss "Shape reconstruction on a varying mesh". IEEE Transactions on Pattern Analysis and Machine Intelligence, 12(4):345-362, 1990.

[16] D. J. Williams and M. Shah "A fast algorithm for active contours and curvature estimation". Computer Vision Graphics Image Processing: Image Undersanding, 55(1):14-26, 1992. 